\title{
Coherence properties of cycling chaos
}

\author{
T. A. Levanova, G. V. Osipov \\ Department of Control Theory, Nizhni Novgorod State University, Gagarin Av. 23, \\ 606950, Nizhni Novgorod, Russia \\ A. Pikovsky \\ Institute for Physics and Astronomy, University of Potsdam, Karl-Liebknecht-Str. 24-25, \\ 14476 Potsdam, Germany
}

\begin{abstract}
Cycling chaos is a heteroclinic connection between several chaotic attractors, at which switching between the chaotic sets occur at growing time intervals. Here we characterize the coherence properties of these switchings, considering nearly periodic regimes that appear close to the cycling chaos due to imperfections or to instability. Using numerical simulations of coupled Lorenz, Roessler, and logistic map models, we show that the coherence is high in the case of imperfection (so that asymptotically the cycling chaos is very regular), while it is low close to instability of the cycling chaos.
\end{abstract}

Keywords: Heteroclinic cycle, Chaos, Coherence

2000 MSC: 37G35, 34D45, 34C37

\section{Introduction}

Heteroclinic cycles have attracted a lot of interest recently. This phenomenon was first introduced by Guckenheimer and Holmes [1, 2]. Heteroclinic cycles are stable regimes of switches between meta-stable states, where these states are observed during longer and longer periods of time, so that the cycle period grows indefinitely. In the phase space of the system, a trajectory passes sequentially through vicinities of saddles, approaching the limiting closed orbit composed of heteroclinic pieces [3, 4]. Physically, heteroclinic cycle appears as a sequential excitation of system's elements [5]. The cases of heteroclinic cycles based on saddle equilibria [6] or saddle cycles [7, 8, 9] and heteroclinic orbits connecting them are well studied. 
An interesting variant of a heteroclinic cycle is cycling chaos, first described by Delltitz et al. [10] and then studied in Refs. [11, 12, 4]. Here the saddle states, which a trajectory approaches in the course of evolution, are chaotic states. In the simplest setup one observes sequential periods of chaotic activity of the participating systems, interrupted by epochs of quiescence. While the internal oscillations are strongly irregular, the switching has a large degree of regularity.

In this paper we study coherence properties of the cycling chaos. We characterize the irregularity of the switchings by the variation of their periods, which is equivalent to the phase diffusion constant, and study how it depends on the parameters. The paper is organized as follows. In Section 2 we introduce three models of cycling chaos. Two of them are based on coupled continuous Lorenz and Rossler oscillators. The third model is a discrete and based on coupled logistic maps. Then we study the statistical properties of these models in Section 3 .

\section{Models of cycling chaos}

All models of cycling chaos that we use below are based on standard models of chaos, interaction of which is organized to ensure cycling. The first model is three coupled Lorenz systems

$$
\begin{aligned}
\dot{x}_{i} & =\sigma\left(y_{i}-x_{i}\right) \\
\dot{y}_{i} & =R_{i} x_{i}-x_{i} z_{i}+\delta \\
\dot{z}_{i} & =-b z_{i}+x_{i} y_{i}+\delta
\end{aligned}
$$

where $i=1,2,3$ and parameters $\sigma=10, b=8 / 3$ are the standard ones. The coupling is via the dependence of parameters $R_{i}$ on the states of the other oscillators:

$$
R_{i}=28\left(1-0.08 D z_{i+1}-0.16 z_{i-1}\right)
$$

where the periodicity in the index is assumed (so that $z_{4} \equiv z_{1}, z_{0} \equiv z_{3}$ ). Parameter $D$, as we now show, is responsible for the transition to cycling. The small parameter $\delta$ describes non-perfectness of the heteroclinic cycle.

The dynamics of system (1) is illustrated in Fig. 1. The panel (a) shows the dynamics of variables $z_{i}$ for $\delta=0$ and $D=0.4$. For these parameters there exist three invariant chaotic sets, where one Lorenz system is active (e.g., $x_{1}, y_{1}, z_{1} \neq 0$ ), while two other vanish (e.g., $x_{i}=y_{i}=z_{i}=0$ for 

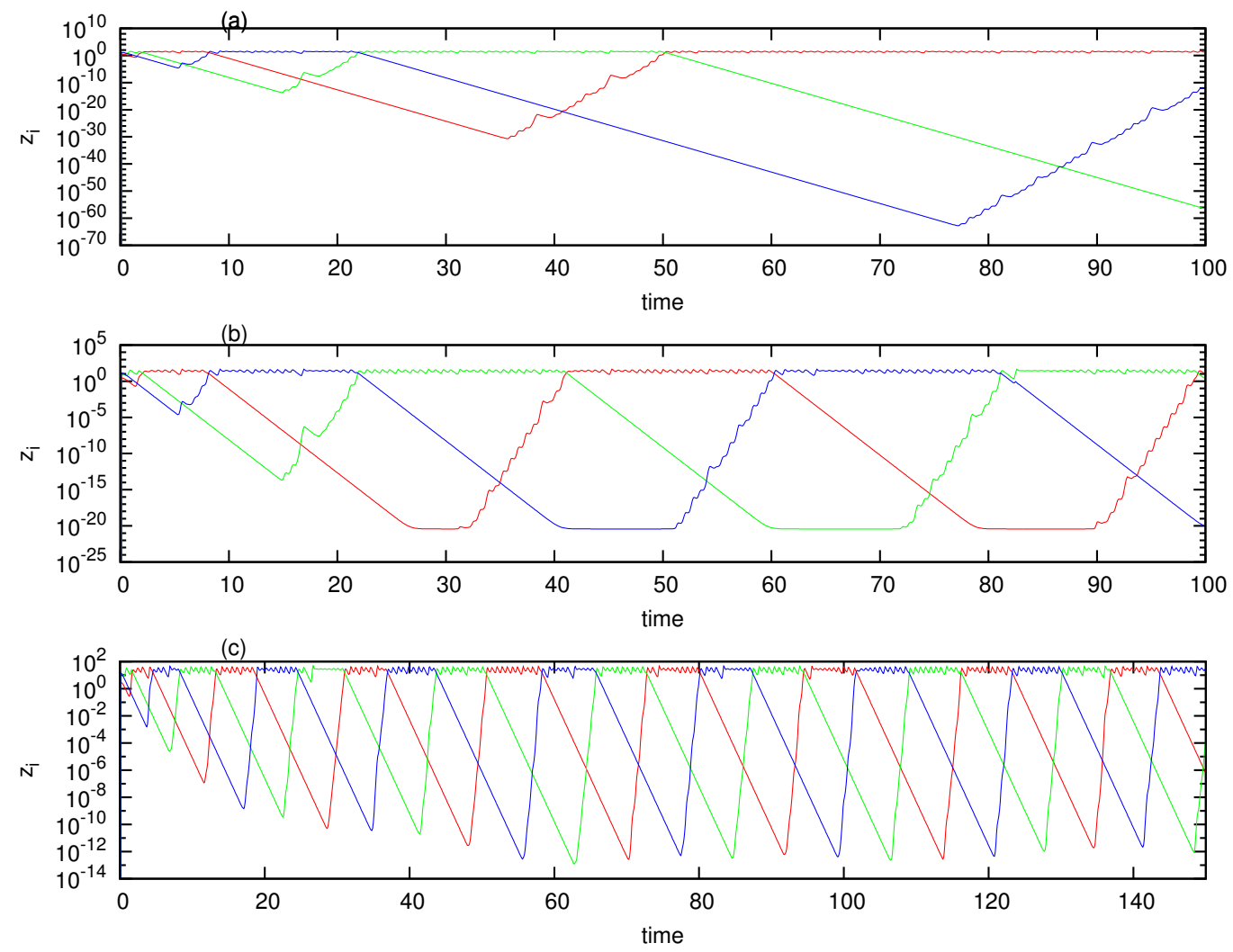

Figure 1: Dynamics of Lorenz model (1). Lines of different colors show time tependence of variables $z_{i}$. (a) $D=0.4, \delta=0$ : cycling chaos. (b) $D=0.4, \delta=10^{-20}$ : imperfect cycling chaos. (c) $D=0.3, \delta=0$ : heteroclinic contour is unstable and a cycle in its vicinity is observed. 
$i=2,3)$. These sets are transversally saddles, forming an attracting heteroclinic cycle. One can see how a trajectory approaches this cycle, staying at each of chaotic sets for longer and longer times. The panel (b) shows the regime for $D=0.4$ and $\delta=10^{-20}$. Now the non-active Lorenz systems do not vanish, but the variables have values $\approx \delta$. Correspondingly, the trajectory approaches a cycling orbit, period of which is bounded from above, tending to infinity as non-perfectness $\delta$ decreases. Finally, in the panel (c) we show the same system for $\delta=0$ and $D=0.3$. At this parameter of coupling the invariant chaotic sets, although present, do not constitute a stable heteroclinic cycle, but because the repelling normal Lyapunov exponent is larger than the attracting one, a regime with a bounded period appears (see [12] for details).

Our goal is to characterize cycles at middle and bottom panels of Fig. 1 statistically. Together with the Lorenz model (1), we consider two other models with similar behavior. A. Palacios [11] has introduced the model of coupled logistic maps (which we generalize by introducing the parameter $\delta$ having the same meaning as above)

$$
\begin{aligned}
& x_{n+1}=\lambda x_{n}\left(1-x_{n}\right)+\alpha x_{n}\left(y_{n}\right)^{0.1}+\delta \\
& y_{n+1}=\lambda y_{n}\left(1-y_{n}\right)+\alpha y_{n}\left(z_{n}\right)^{0.1}+\delta \\
& z_{n+1}=\lambda z_{n}\left(1-z_{n}\right)+\alpha z_{n}\left(x_{n}\right)^{0.1}+\delta
\end{aligned}
$$

Here we fix, following [11], $\lambda=3.8$ and consider $\delta$ as a parameter of imprefection, and $\alpha$ as a parameter responsible for the lost of stability of the heteroclinic cycle.

Furthermore, we consider three coupled (similar to the coupling in (1)) Roessler systems

$$
\begin{aligned}
\dot{x}_{i} & =-y_{i}-z_{i}+\delta \\
\dot{y}_{i} & =a_{i} y_{i}+x_{i}+\delta \\
\dot{z}_{i} & =z_{i}\left(x_{i}-10\right)+0.02 * x_{i}
\end{aligned}
$$

with $a_{i}=0.15\left(1-0.006 D\left(x_{i+1}^{2}+y_{i+1}^{2}\right)-0.012\left(x_{i-1}^{2}+y_{i-1}^{2}\right)\right)$. Parameters $\delta$ and $D$ have the same meaning as for the Lorenz systems (1).

\section{Statistical properties of cycles}

Our main aim is to describe the nearly-heteroclinic cycling chaos like in Fig. 11 statistically. One can see from this figure, that the main macroscopic 
irregularity is in different lengths of epochs where one of three participating subsystems dominate. To characterize this we performed long runs to get the local periods of cycles $T_{k}, k=1, \ldots, 10^{6}$, and from these data we calculated the mean period $\langle T\rangle$ and the standard deviation $\Delta T=\left\langle(T-\langle T\rangle)^{2}\right\rangle^{1 / 2}$. The ratio $\gamma=\frac{\Delta T}{\langle T\rangle}$ is a dimensionless quantity characterizing the coherence of the cycle, it is proportional to the phase diffusion constant normalized by the period.

First we present the results for the cycles appearing due to the imperfectness parameter $\delta$. In Fig. 2 we show the dependence of $\langle T\rangle$ on $\log \delta$ for the models considered. As one can expect, the dependence is linear.

(a)

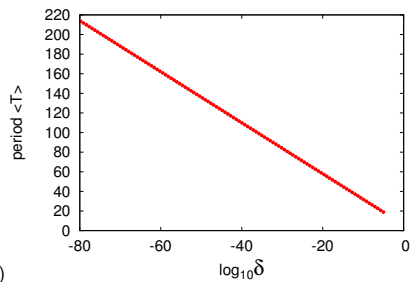

(b)

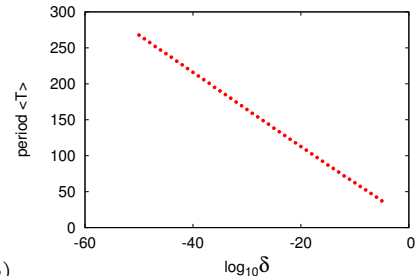

Figure 2: Dependencies of the average period on the parameter $\delta$ : (a) Lorenz model (1) with $D=0.4$, (b) Logistic map model (3) with $\alpha=-3.72$, (c) Roessler model (4) with $D=0.8$
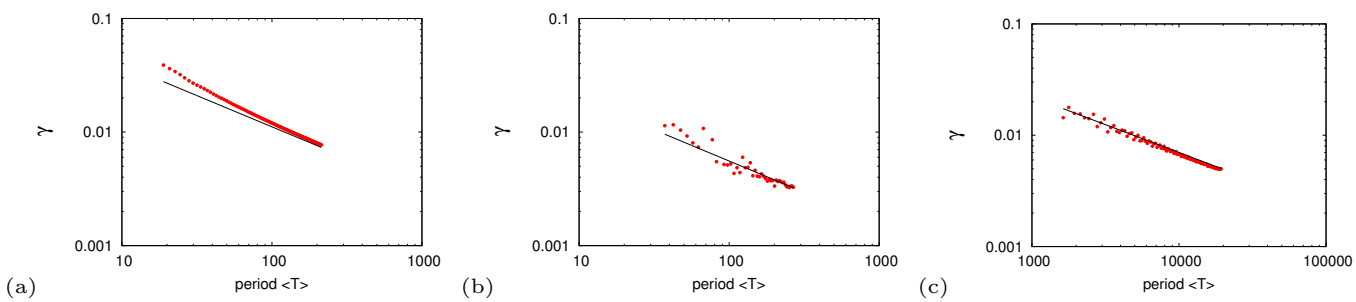

Figure 3: Dependencies of the cycle coherence $\gamma$ on the average period for the same parameters as in Fig. 22 (a) Lorenz model (1), (b) Logistic map model (3), (c) Roessler model (4). The slopes of the lines in (a),(b) are 0.55 , in (c) the slope is 0.5 .

The coherence parameter $\gamma$ is presented in Fig. 3. One can see that in all cases the coherence improves as the period of the cycle increases, roughly $\gamma=\frac{\Delta T}{\langle T\rangle} \sim\langle T\rangle^{-1 / 2}$. This indicates that asymptotically for long cycles the coherence is large: chaotic epochs do not contribute significantly to the phase diffusion.

In Figs. 4,5 we report similar calculations for the second type of nearlyheteroclinic cycle, presented in Fig. 1(c). Figure 4 shows the dependencies of 

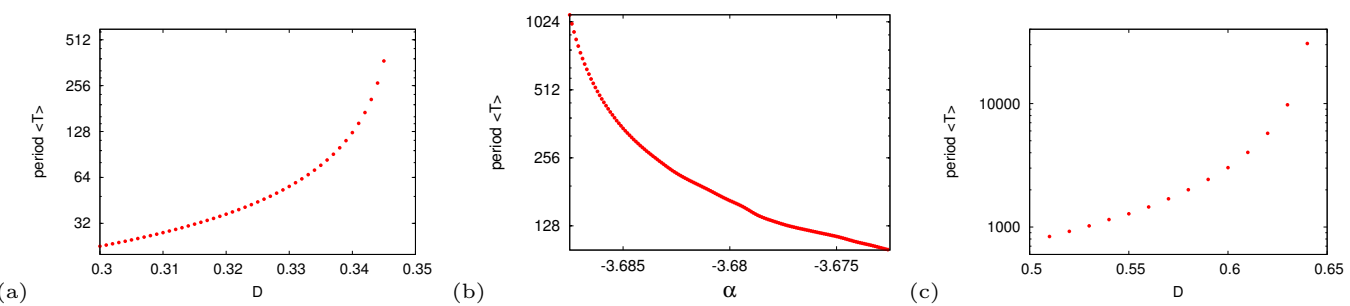

Figure 4: Variation of the chaotic cycle period near the transition to a chaotic homoclinic cycle, for $\delta=0$. (a): Lorenz model, dpendence on parameter $D$. (b): Logistic map model, dependence on parameter $\alpha$. (c): Roessler model, dependence on parameter $D$.
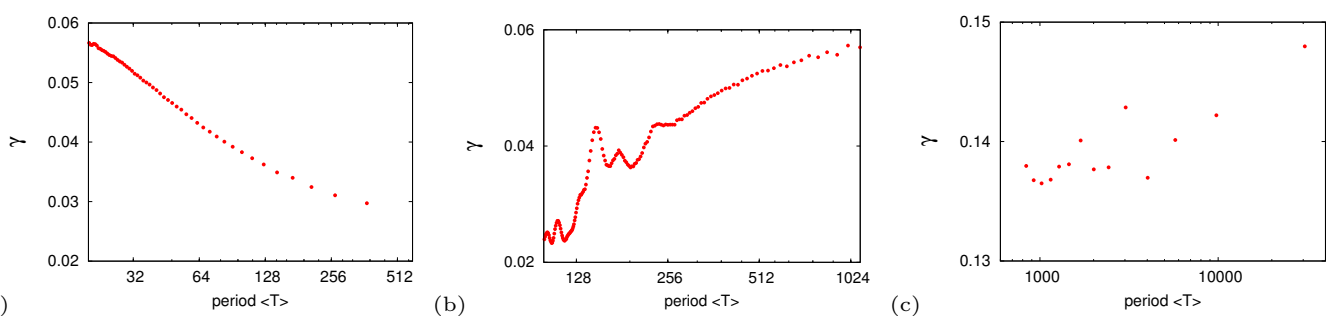

Figure 5: Dependencies of the cycle coherence $\gamma$ on the average period for the data presented in Fig. 4. (a) Lorenz model, (b) Logistic map model, (c) Roessler model. Notice that contrary to the Fig. 3 , here on all panels the vertical axis is linear and not logarithmic.

the average period on the coupling parameters ( $D$ for Lorenz and Roessler models, $\alpha$ for the logistic maps). Near the criticality the period grows, although the precise law of this growth is not easy to follow, because the critical values of coupling are known only approximately. The coherence parameter is presented in Fig. 5, again as a function of the period. One can see that the behavior for the three models is different: for the Lorenz model the coherence improves with the period, while for the logistic maap model it decreases; for the Roessler model no significant changes are observed. In all three cases the coherence parameter $\gamma=\frac{\Delta T}{\langle T\rangle}$ does not appear to follow a power-law, rather it seems that it remains bounded as $\langle T\rangle \rightarrow \infty$. (For the only candidate for a power-law, the Lorenz system, we plotted the data of Fig. 5 in a log-log representation and observed that it does not follow a power-law, but the slope decreases from $\approx-0.3$ to $\approx-0.15$, with a tendency to saturation of the coherence.) The results of the simulations for all three models show that the coherence of the long cycles close to the transition is rather weak.

The difference in the behavior of the coherence parameter can be explained as follows. In the case of the imperfect heteroclinic cycle $(\delta \neq 0$, 
Figs. 22 3) the minimal level of activity is fixed by the value of $\delta$, the period of the cycle is basically determined by the time needed to reach the activity starting from $\delta$, and the variations of this time are relatively small. In the case where the finite cycle appears from the stable heteroclinic one at the critical coupling, where the negative transverse Lyapunov exponent becomes equal to the positive one, the minimal level of activity is determined by the difference of these exponents. Because the Lyapunov exponents in chaos fluctuate, this minimal level experiences significant fluctuations as well. This can be clearly seen as fluctuations of minima in Fig. 1(c). Thus, the time needed to reach activity from the minimal level fluctuates as well, which results in relatively weak coherence.

\section{Conclusion}

Cycling chaos is an interesting example of nontrivial dynamics "on top" of chaos. In other examples, such as chaos-induced diffusion [13, 14], chaotic motion enters explicitely. Here in the ideal case chaos lives on the invariant manifolds of three participating oscillators, and in perturbed case only finite epochs of chaotic dynamics are observed. This makes analytical calculations of statistical properties of cycling chaos a challenging problem for the future, therefore in this communication we followed a numerical approach. We simulated three models, two are coupled continuous-time chaotic systems (Lorenz and Roessler models), and one is coupled logistic maps. Our approach was not to characterize cycling chaos, as it is a nonstationary process, but to characterize the coherence of the nearly periodic oscillations that appear if the cycling chaos is disturbed, and to check the properties of the coherence as the cycle approaches pure heteroclinic cycling chaos, and its average period grows. In all cases we observed a clear difference between the situations when the cycling chaos was destroyed by an imperfection in the equations (the chaotic manifolds are no more invariant ones), and when it becomes unstable as the instability of the manifolds becomes stronger than the attraction to them. In the former case the coherence is high and the deviations from the average period follow the power law, growing roughly as a square root of the period. This means that the relative coherence increases with the period of the cycle. When the cycling chaos loses its stability, we observe that the deviations are roughly proportional to the period, what means that the relative coherence remains finite. The properties of cycle coherence are important, e.g., for synchronizability of the cycling chaos, what is the subject 
of future work.

Acknowledgements We would like to thank Russian Federal Program Scientific and Scientific-Educational Brainpower of Innovative Russia for 2009-2013 (contract No.14.B37.21.0863). A.P. thanks Nizhni Novgorod State University for warm hospitality.

\section{References}

[1] J. Guckenheimer, P. Holmes, Structurally stable heteroclinic cycles, Math. Proc. Camb. Phil. Soc. 103 (1988) 189-192.

[2] D. Armbruster, J. Guckenheimer, P. J. Holmes, Heteroclinic cycles and modulated travelling waves in systems with O2 symmetry., Physica D 29 (1988) 257-282.

[3] P. Ashwin, P. Chossat, Attractors for robust heteroclinic cycles with continua of connections, J. Nonlin. Sci. 8 (1998) 103-129.

[4] P. Ashwin, M. Field, Heteroclinic networks in coupled cell systems, Arch. Rational Mech. Anal. 148 (1999) 107-143.

[5] V. S. Afraimovich, V. P. Zhigulin, M. I. Rabinovich, On the origin of reproducible sequential activity in neural circuits, Chaos 14 (4) (2004) $1123-1129$.

[6] M. Krupa, Robust heteroclinic cycles, J. Nonlinear Sci. 7 (1997) 129176.

[7] M. A. Komarov, G. V. Osipov, J. A. K. Suykens, equentially activated groups in neural networks, EPL 86 (2009) 60006.

[8] A. O. Mikhaylov, M. A. Komarov, T. A. Levanova, G. V. Osipov, Sequential switching activity in ensembles of inhibitory coupled oscillators, EPL 101 (2013) 20009.

[9] M. A. Komarov, G. V. Osipov, C. S. Zhou, Heteroclinic contours in oscillatory ensembles, Phys. Rev. E 87 (2013) 022909.

[10] M. Dellnitz, M. Field, M. Golubitsky, A. Hohmann, J. Ma, Cycling chaos, IEEE Trans. Circuits Systems 42 (10) (1995) 3821-823. 
[11] A. Palacios, Cycling chaos in one-dimensional coupled iterated maps, Int. J. Bif. Chaos 12 (8) (2002) 1859-1868.

[12] P. Ashwin, Cycles homoclinic to chaotic sets; robustness and resonance, Chaos 7 (1997) 207-220.

[13] T. Geisel, J. Nierwetberg, Onset of diffusion and universal scaling in chaotic systems, Phys. Rev. Lett 48 (1) (1982) 7-10.

[14] W. Just, H. Kantz, C. Rödenbeck, M. Helm, Stochastic modelling: Replacing fast degrees of freedom by stochastic processes, J. Phys. A: Math., Gen. 34 (2001) 3199. 